\title{
K6PC-5 Activates SphK1-Nrf2 Signaling to Protect Neuronal Cells from Oxygen Glucose Deprivation/Re-Oxygenation
}

\author{
Hua Liu ${ }^{\mathrm{a}} \quad$ Zhiqing Zhang ${ }^{\mathrm{b}} \quad{\text { Min } X u^{c}}^{\mathrm{R}}$ Rong $\mathrm{Xu}^{\mathrm{d}}$ Zhichun Wang ${ }^{\mathrm{e}}$ Guangfu Di ${ }^{\mathrm{e}}$ \\ aDepartment of Neurosurgery, The First People's Hospital of Kunshan, Jiangsu University, Suzhou, \\ bInstitute of Neuroscience, Soochow University, Suzhou, 'Department of Neurosurgery, Kunshan \\ Hospital of Traditional Chinese Medicine, Kunshan Affiliated Hospital, Suzhou, dDepartment of \\ Pediatric surgery, The First People's Hospital of Kunshan, Jiangsu University, Suzhou, eDepartment of \\ Neurosurgery, Yijishan Hospital, Wannan Medical College, Wuhu, China
}

\section{Key Words}

Neuron $\cdot \mathrm{K} 6 \mathrm{PC}-5 \cdot \mathrm{SphK} 1 \cdot \mathrm{Nrf2} \cdot$ Oxidative stress

\begin{abstract}
Background/Aims: New strategies are required to combat neuronal ischemia-reperfusion injuries. K6PC-5 is a novel sphingosine kinase 1 (SphK1) activator whose potential activity in neuronal cells has not yet been tested. Methods: Cell survival and necrosis were assessed with a Cell Counting Kit- 8 assay and lactate dehydrogenase release assay, respectively. Mitochondrial depolarization was tested by a JC-1 dye assay. Expression levels of nuclear factor erythroid 2-related factor 2 (Nrf2) signaling components were examined by quantitative real-timePCR and western blotting. Results: K6PC-5 protected SH-SY5Y neuronal cells and primary murine hippocampal neurons from oxygen glucose deprivation/re-oxygenation (OGDR). K6PC5 activated SphK1, and SphK1 knockdown by targeted short hairpin RNA (shRNA) almost completely abolished K6PC-5-induced neuronal cell protection. Further work showed that K6PC-5 inhibited OGDR-induced programmed necrosis in neuronal cells. Importantly, K6PC5 activated Nrf2 signaling, which is downstream of SphK1. Silencing of Nrf2 by targeted shRNA almost completely nullified K6PC-5-mediated neuronal cell protection against OGDR. Conclusion: K6PC-5 activates SphK1-Nrf2 signaling to protect neuronal cells from OGDR. K6PC-5 might be a promising neuroprotective strategy for ischemia-reperfusion injuries.
\end{abstract}




\section{Introduction}

During the pathogenesis of stroke, ischemia-reperfusion can severely damage neurons $[1,2]$. Ischemia-reperfusion injury can be mimicked in cultured neuronal cells using oxygen glucose deprivation and re-oxygenation (OGDR) [3-6]. OGDR-induced neuronal cell death is mediated by oxidative stress [5,7], with sustained oxygen glucose deprivation (OGD; often over $1 \mathrm{~h}$ ) disrupting mitochondrial functions. After re-oxygenation, massive amounts of reactive oxygen species (ROS) are produced, causing oxidative stress, lipid peroxidation, DNA damage, and neuronal cell death [5, 7].

Evidence shows that the mitochondrial permeability transition pore (mPTP) mediates neuronal cell death after oxidative stress [8]. The mPTP is primarily composed of two structural components, the adenine nucleotide translocase-1 (ANT-1) and the voltage-dependent anion transporter (VDAC), as well as the regulatory component, cyclophilin D (Cyp-D) [911]. Cyp-D and ANT-1 are found in the inner mitochondrial membrane, whereas VDAC is in the outer mitochondrial membrane [9-11]. Oxidative stress can trigger the translocation of p53 to mitochondria, where it associates with Cyp-D to cause a conformational change in ANT-1, mPTP opening, and eventually cell necrosis (but not apoptosis) [9, 11].

Sphingolipid metabolites, including ceramide, sphingosine, and sphingosine-1phosphate (S1P), are important signaling determinants of cell survival/death [12]. Sphingosine kinase 1 (SphK1) phosphorylates sphingosine to S1P, which depletes prodeath sphingosine and ceramide but increases the production of pro-survival S1P [13, 14]. Thus, SphK1 activation promotes cell survival $[13,14]$. Recent studies have led to the development of a novel, specific, and highly potent SphK1 activator, named K6PC-5 ( $N-(1$, 3-dihydroxyisopropyl)-2-hexyl-3-oxo-decanamide) $[15,16]$. Here, we show that activation of SphK1 by K6PC-5 protects neuronal cells from OGDR.

\section{Materials and Methods}

\section{Reagents}

K6PC-5 was provided by Dr. Fei [17]. Puromycin and cell culture reagents were obtained from SigmaAldrich (St. Louis, MO). Antibodies were all provided by Santa Cruz Biotechnology (Santa Cruz, CA) and Cell Signaling Technology (Shanghai, China).

\section{SH-SY5Y cell culture}

Human neuronal SH-SY5Y cells were provided by the iBS Cell Bank of Fudan University (Shanghai, China). Cells were maintained in Dulbecco's modified Eagle's medium (DMEM) plus 10\% fetal bovine serum and the necessary antibiotics. Before OGDR treatment, SH-SY5Y cells were cultured for 5 days with $10 \mu \mathrm{M}$ retinoic acid (RA) in DMEM plus 10\% FBS, 2 mM glutamine, and necessary antibiotics, followed by another 5 days culture in serum-free DMEM with BDNF (brain-derived neurotrophic factor, $50 \mathrm{ng} / \mathrm{mL}$ ) and glutamine $(2 \mathrm{mM})$.

\section{Primary culture of murine hippocampal neurons}

Primary hippocampal neurons were derived from the CA1 hippocampus of C57 mouse E12-E14 embryos. CA1 neurons were plated in poly-lysine-coated 48-well plates at a density of $1 \times 10^{5}$ cells/well in neurobasal medium plus 2\% B27, $500 \mu \mathrm{M}$ L-glutamine, $20 \mathrm{ng} / \mathrm{mL}$ trichostatin A (TSA) and antibiotics (P/S). At day8, over $95 \%$ of cells were neurons. All animal studies were performed in accordance with the ethical treatment standards of the Institutional Animal Care and Use Committee of Jiangsu University.

\section{Cell survival, apoptosis and death assay}

The Cell Counting Kit-8 (CCK-8) assay (Dojindo Laboratories, Kumamoto, Japan) was performed to examine the viability of neuronal cells. The CCK optical density (OD) at $450 \mathrm{~nm}$ was recorded. Cell necrosis was tested via examination of the release of lactate dehydrogenase (LDH) to the conditioned medium using a two-step enzymatic reaction LDH assay kit (Takara, Tokyo, Japan). The LDH level in the medium was 


\section{Cellular Physiology Cell Physiol Biochem 2018;51:1908-1920 \begin{tabular}{l|l|l} 
and Biochemistry & $\begin{array}{l}\text { DOI: 10.1159/000495716 } \\
\text { Published online: } 1 \text { December } 2018\end{array}$ & $\begin{array}{l}\text { @ 2018 The Author(s). Published by S. Karger AG, Basel } \\
\text { www.karger.com/cpb }\end{array}$ \\
\hline
\end{tabular}}

Liu et al.: K6PC-5 Activates SphK1-Nrf2 Signaling

normalized to total LDH (medium LDH plus cellular LDH). The TUNEL assay and the caspase-3 activity were described in detail elsewhere [18].

$O G D R$

The OGDR procedure was as described previously [3]. Briefly, neuronal cells were first placed in an airtight chamber and equilibrated for 10 min with a continuous flux of gas $\left(95 \% \mathrm{~N}_{2} / 5 \% \mathrm{CO}_{2}\right)$. The chamber was sealed and placed in an incubator for an additional $4 \mathrm{~h}$ of OGD. Cells were then re-oxygenated in the normal aerobic environment. Mock cells were placed in norm-oxygenated DMEM containing glucose.

\section{Mitochondrial immunoprecipitation}

The mitochondrial immunoprecipitation (mito-IP) protocol has been detailed previously [3, 19, 20]. Briefly, $10 \times 10^{6} \mathrm{SH}-\mathrm{SY} 5 Y$ cells per treatment were homogenized by lysis buffer A (250mM sucrose, $20 \mathrm{mM}$ HEPES, $10 \mathrm{mM} \mathrm{KCl}, 1.5 \mathrm{mM} \mathrm{MgCl}{ }_{2}, 1 \mathrm{mM}$ EDTA, $1 \mathrm{mM}$ EGTA, and $1 \mathrm{mM}$ dithiothreitol). After centrifugation, the cell pellets were resuspended in buffer B (1mL buffer A containing $10 \mu \mathrm{L} \mathrm{NP-40)}$ and collected as the mitochondrial fraction. The pre-cleared mitochondrial lysates (600 $\mu \mathrm{g}$ per sample) were incubated overnight with anti-Cyp-D antibody $[19,21]$. The mitochondrial immune complexes were then captured by protein A/G beads (Sigma-Aldrich). Cyp-D-p53 associations were then tested by western blotting.

\section{Western blot}

Neuronal cells were first incubated with RIPA lysis buffer (Biyuntian, Wuxi, China). Forty micrograms of lysate proteins per lane were separated on 10-12\% sodium dodecyl sulfate-polyacrylamide gel electrophoresis (PAGE) gels, then transferred to polyvinylidene difluoride (PVDF) membrane (Millipore, Suzhou, China)[22]. After blocking, the blots were incubated with the appropriate primary and secondary antibodies. Enhanced chemiluminescence (ECL) reagents (Pierce, Suzhou, China) were applied to visualize the targeted protein bands (based on the molecular weight). The total gray value of each band was quantified using Image J software (National Institutes of Health, Bethesda, MD).

\section{Mitochondrial depolarization assay}

JC-1 dye was used to test for mitochondrial membrane potential changes [23]. After mitochondrial depolarization, JC-1 red aggregates form green monomers [24]. Briefly, neuronal cells were stained with JC-1 (10 $\mu \mathrm{g} / \mathrm{mL}$; Invitrogen, Shanghai, China) for $15 \mathrm{~min}$ at room temperature. JC-1 green intensity was examined immediately at $550 \mathrm{~nm}$ via a fluorescence spectrofluorometer.

\section{Quantitative real-time PCR assay}

Cellular RNA was extracted with TRIzol reagents (Promega, Shanghai, China). A SYBR Green PCR kit (Applied Biosystems, Suzhou, China) was used for reverse transcription, and theABI Prism7600 Fast Real-Time PCR system was used for the quantitative real-time PCR (qPCR) assay. Melting curve analysis was performed to calculate the product melting temperature. Glyceraldehyde-3-phosphatedehydrogenase $(G A P D H)$ was always tested as the reference gene. The $2^{-\Delta \Delta C t}$ method was used to quantify the relative mRNA expression. The mRNA primers for SphK1 were as described previously [25]. The mRNA primers for nuclear factor erythroid 2-related factor 2 (Nrf2), heme oxygenase 1 (HO1), NAD(P)H quinone oxidoreductase 1 (NQO1), $\gamma$-glutamylcysteine synthetase catalytic subunit (GCLC), and GAPDH were also described [26, 27].

\section{Superoxide assay}

The cellular superoxide level wastested with a superoxide assay kit (Beyotime Institute of Biotechnology, Wuxi, China) according to the manufacturer's protocol. Briefly, neuronal cells were cultured in 6-well plates at a density of $3 \times 10^{5}$ cells/well. After the applied treatment, the superoxide detection reagent $(200 \mu \mathrm{L} /$ well) was added at $37^{\circ} \mathrm{C}$ for $20 \mathrm{~min}$. The absorbance was recorded at $450 \mathrm{~nm}$ in a 96 -well plate reader.

SphK1 activity assay

After the applied treatment, $100 \mu \mathrm{g}$ lysates per treatment was incubated with $25 \mu \mathrm{M}$ D-erythrosphingosine dissolved in $0.1 \%$ Triton $\mathrm{X}-100,2 \mathrm{mM}$ ATP, and $\left[{ }^{\gamma-32} \mathrm{P}\right]$ ATP for $30 \mathrm{~min}$ at $37^{\circ} \mathrm{C}$ [28]. The reaction was terminated via the addition of $20 \mu \mathrm{L}$ of $\mathrm{HCl}$, plus $800 \mu \mathrm{L}$ of chloroform/methanol $/ \mathrm{HCl}$ $(100: 200: 1, v / v)$. After vortexing, $250 \mu \mathrm{L}$ of chloroform and $250 \mu \mathrm{L}$ of $\mathrm{KCl}$ were added, and the phases were 
separated by centrifugation. The organic layer was dried and resuspended in chloroform/methanol/HCl $37 \%(100: 100: 0.2, \mathrm{v} / \mathrm{v})$. Lipids were resolved on silica TLC plates in 1-butanol/acetic acid/water (3:1:1, $\mathrm{v} / \mathrm{v}$ ). Labeled S1P spots were visualized by autoradiography and quantified by scraping and counting in a scintillation counter. SphK1 activity was evaluated as $\mathrm{pmol} / \mathrm{h} / \mathrm{g}$ protein.

\section{Short hairpin RNA}

Two lentiviral SphK1 short hairpin RNAs (shRNAs) were provided by Dr. Qin from Nanjing University of TCM [28]. Nrf2 lentiviral shRNA and the scrambled control shRNA were provided by Dr. Jiang from Nanjing Medical University [26]. shRNA lentivirus (10 $\mu \mathrm{L} / \mathrm{mL}$ medium per well) was added to neuronal cells for 24 h. Stable cells were selected by puromycin $(2.5 \mu \mathrm{g} / \mathrm{mL}$; Sigma-Aldrich) for another 6 days. Expression of the targeted protein was determined by western blot.

\section{Statistical analysis}

The results are expressed as the mean \pm standard deviation (SD). Statistical analysis among different groups was performed via one-way analysis of variance with Scheffe's test using SPSS18.0 software (SPSS Inc., Chicago, IL). Experiments were repeated at least three times and consistent results were always obtained.

\section{Results}

K6PC-5 protects neuronal cells from OGDR

SH-SY5Y, the established human neuronal cells $[29,30]$, were treated with different concentrations of K6PC-5, from 2.5 to $100 \mu \mathrm{M}$. The CCK-8 assay results, shown in Fig. 1A, revealed that K6PC-5 was generally safe to SH-SY5Y cells, except at very high concentrations $(100 \mu \mathrm{M})$. The latter induced a reduction in CCK-8 OD (Fig. 1A). LDH release to the medium is a characteristic marker of cell necrosis. Treatment of SH-SY5Y cells with $100 \mu \mathrm{M}$ of K6PC-5 induced a significant LDH release (Fig. 1B). At other tested concentrations $(2.5,10$, and 25 $\mu \mathrm{M}$ ), K6PC-5 failed to induce cell death (Fig. 1B). Recent studies have used K6PC-5 at $10 \mu \mathrm{M}$ in vitro $[17,31]$. This concentration was thus applied for the remaining experiments.

SH-SY5Y cells were subjected to OGD for different periods, from 2 to $6 \mathrm{~h}$. Afterward, the cells were maintained in the normal aerobic environment (re-oxygenation, OGDR) for another $24 \mathrm{~h}$. The CCK-8 assay results confirmed that OGD for 4 and $6 \mathrm{~h}$ significantly inhibited SH-SY5Y cell viability (Fig. 1C). Co-treatment with K6PC-5 $(10 \mu \mathrm{M})$ attenuated the reduction in viability induced by OGDR (Fig. 1C). OGDR-induced LDH release was also significantly inhibited by K6PC-5 (Fig. 1D). These results indicate that K6PC-5 protects SH-SY5Y cells from OGDR.

The effect of the novel SphK1 activator on primary murine hippocampal neurons was next tested. The results showed that K6PC-5 $(10 \mu \mathrm{M})$ co-treatment of primary neurons dramatically alleviated the OGDR ( 4 h OGD deprivation, $24 \mathrm{~h}$ re-oxygenation)-induced viability reduction (CCK-8 OD decrease, Fig. 1E) and cell necrosis (LDH release, Fig. 1F). These results again suggest that K6PC-5 protects neuronal cells from OGDR.

\section{K6PC-5-mediated neuroprotection against OGDR requires SphK1}

SphK1 activity and expression in K6PC-5-treated neuronal cells were tested next. K6PC5 dose-dependently increased SphK1 activity in SH-SY5Y cells (Fig. 2A) without affecting SphK1 protein expression (Fig. 2B). Conversely, OGDR induced SphK1 expression in SH-SY5Y cells (Fig. 2C and D). SphK1Mrna (Fig. 2C) and protein (Fig. 2D) levels were increased by OGDR treatment.

To test the link between SphK1 activation and K6PC-5-mediated neuroprotection, the shRNA method was applied to knockdown SphK1. Two different lentiviral shRNAs against non-overlapping sequences (Seq-a/-b) of SphK1 were provided by Dr. Qin [28]. Each of the SphK1 lentiviral shRNAs led to dramatic downregulation of SphK1 in OGDR-treated SHSY5Y cells (Fig. 2E and F). SphK1mRNA (Fig. 2E) and protein (Fig. 2F) levels were largely 


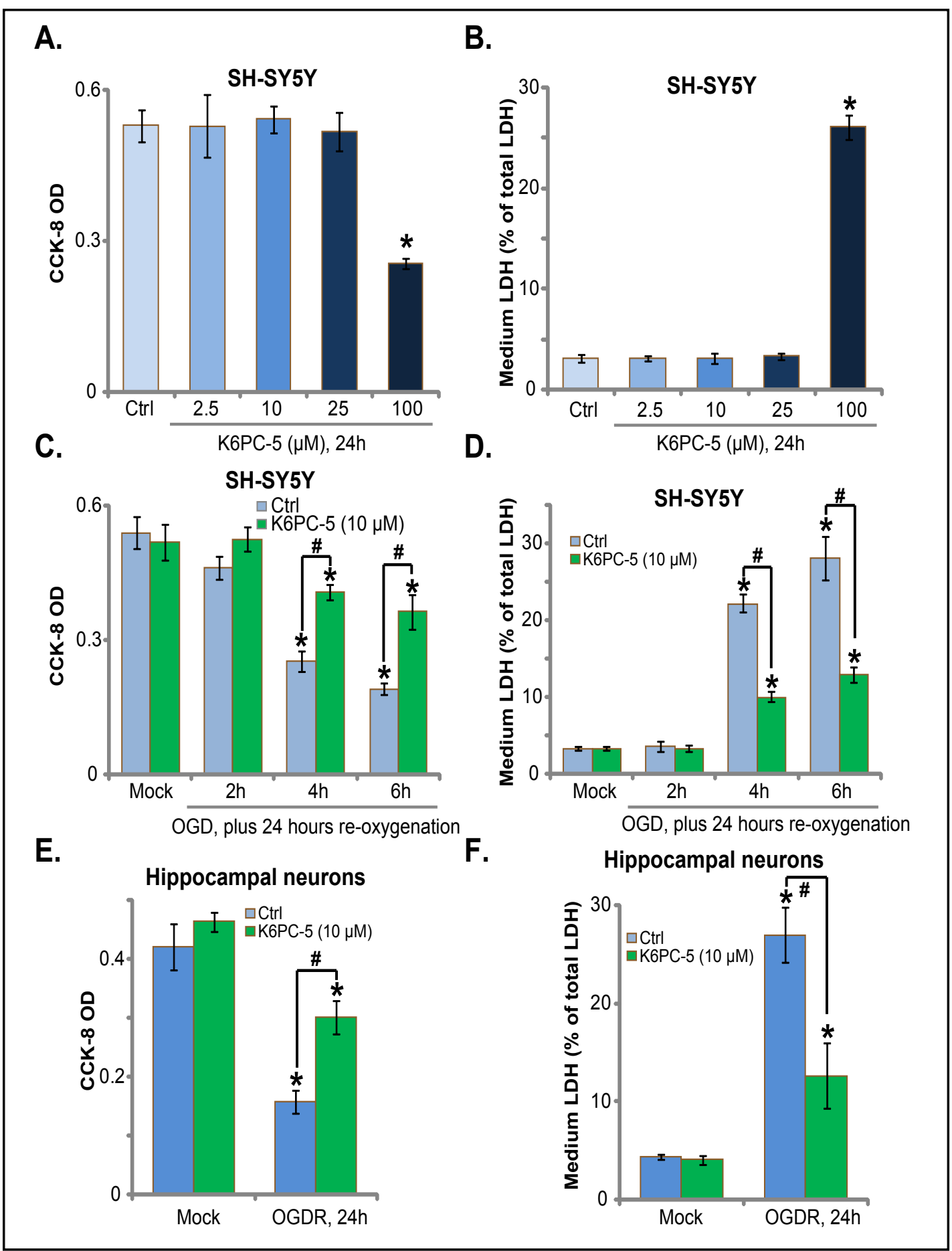

Fig. 1. K6PC-5 protects neuronal cells from OGDR. SH-SY5Y cells (A-D) or primary murine hippocampal neurons ( $\mathrm{E}$ and F) were treated with the indicated concentrations of K6PC-5, with or without OGD; cells were further cultured in a normal aerobic environment (re-oxygenation, OGDR) for an additional $24 \mathrm{~h}$. Cell viability was tested by a CCK-8 assay (A, C, and E), whereas cell necrosis was analyzed by an LDH release assay (B, D, and F). Ctrl indicates the untreated control group (same for all figures). Mock indicates the mock treatment group (no OGDR, regular medium) (same for all figures). Bars indicate the mean \pm SD $(n=5)$. ${ }^{*} \mathrm{p}<0.05$ vs. Ctrl. ${ }^{*} \mathrm{p}<0.05$ vs. OGDR only. Each experiment was repeated four times and similar results were obtained. 

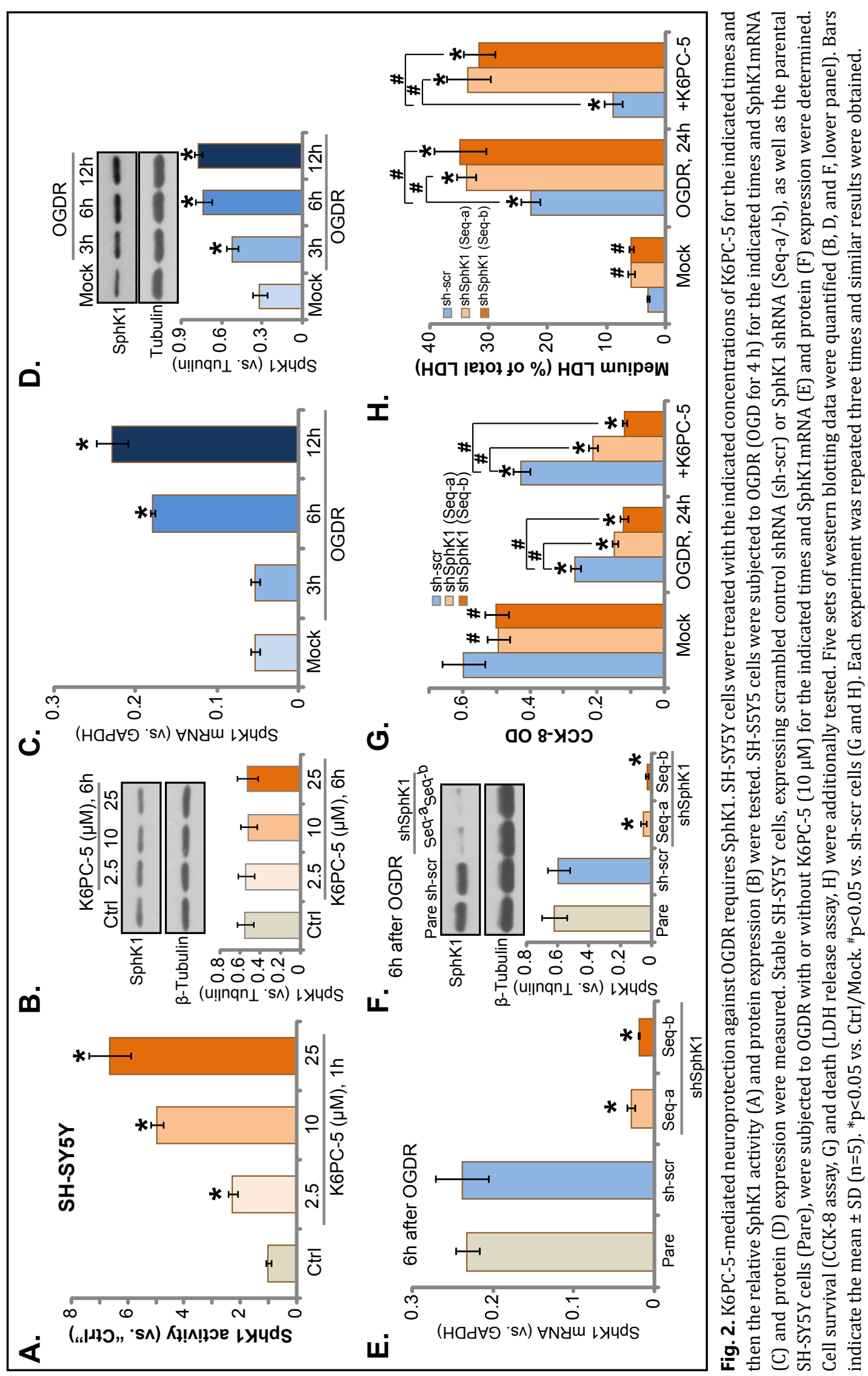


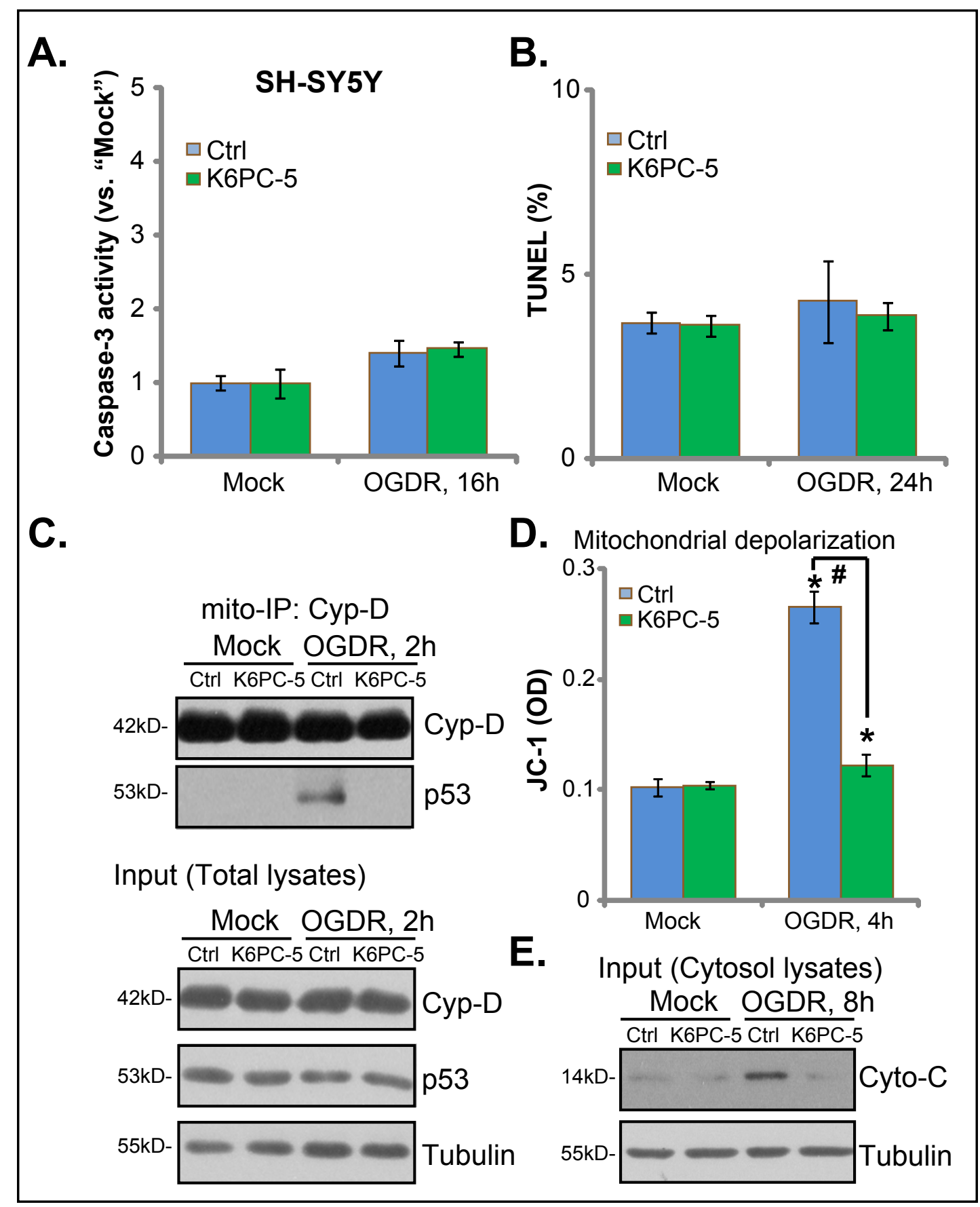

Fig. 3. K6PC-5 inhibits OGDR-induced programmed necrosis in neuronal cells. SH-SY5Y cells were subjected to OGDR with or without K6PC-5 (10 $\mu \mathrm{M})$ for the indicated times and he caspase-3 activity (A), nuclear TUNEL percentage (B), mitochondrial p53-Cyp-D association (C, "mito-IP"), mitochondrial depolarization (JC-1 dye assay, D), and expression of the listed proteins (C, "Input", and E) were tested by the appropriate assays. Bars indicate the mean $\pm \operatorname{SD}(n=5) .{ }^{*} \mathrm{p}<0.05$ vs. Mock. ${ }^{*} \mathrm{p}<0.05$. Each experiment was repeated four times and similar results were obtained. 
inhibited. Importantly, OGDR-induced cytotoxicity (CCK-8 OD reduction and LDH release) was increased in SH-SY5Y cells treated with SphK1 shRNA (Fig. 2G and H). Furthermore, K6PC-5-mediated neuroprotection against OGDR was almost completely abolished in SphK1silenced cells (Fig. 2G and H). Based on these results, we propose that SphK1 is important to combat OGDR. K6PC-5 activated SphK1 to protect SH-SYRY cells from OGDR. The scrambled control shRNA (sh-scr), as expected, did not affect SphK1 expression (Fig. 2E and F) nor OGDR-induced cytotoxicity (Fig. 2G and H).

K6PC-5 inhibits OGDR-induced programmed necrosis in neuronal cells

Studies have shown that OGDR mainly induces cell programmed necrosis (not apoptosis $[5,19,32])$. We showed that OGDR failed to increase caspase-3 activity (Fig. 3A) or nuclear terminal deoxynucleotidyl transferase dUTP nick end labeling (TUNEL) staining percentage (Fig. 3B) in SH-SY5Y cells. OGDR induces p53 translocation to mitochondria, where it associates with Cyp-D[19, 32]. The mitochondrial p53-Cyp-D association then triggers mitochondrial depolarization, mPTP opening, cytochrome $\mathrm{C}$ (Cyto-C) release, and eventually cell necrosis $[19,32]$. Here, OGDR treatment of SH-SY5Y cells induced the mitochondrial p53-Cyp-D association (Fig. 3C), mitochondrial depolarization (JC-1 OD increase, Fig. 3D), and Cyto-C release (Fig. 3E). Importantly, such effects of OGDR were significantly attenuated by K6PC-5 (Fig. 3C-E). p53 and Cyp-D expression levels in total cell lysates were unaffected (Fig. 3C, "Input"). These results suggest that K6PC-5-induced neuronal cell protection against OGDR might occur through inhibition of the programmed necrosis pathway.

\section{K6PC-5 activates Nrf2 signaling in neuronal cells}

Nrf2 disassociates from its inhibitor protein Keap1, causing Nrf2 stabilization and nuclear translocation. Nrf2 then binds to antioxidant response element (ARE) in the nucleus, mediating the transcription and expression of several key antioxidant genes, including NQ01, HO1 and GCLC [33-36]. Here, we showed that the mRNA expression levels of the Nrf2dependent genes NQ01 (Fig. 4A), HO1 (Fig. 4B), and GCLC (Fig. 4C) were significantly elevated after K6PC-5 treatment of SH-SY5Y cells. However, the Nrf2 mRNA level was unchanged (Fig. 4D). The protein expression levels of NQ01, H01, and GCLC were also increased by K6PC5 in SH-SY5Y cells (Fig. 4E). Additionally, the Nrf2 protein level was increased by K6PC-5 treatment (Fig. 4E), indicating Nrf2 protein stabilization. The latter is an essential step forNrf2 nuclear translocation and activation [33-36].

Next, we tested whether SphK1 is required for K6PC-5-induced Nrf2 activation. The qPCR assay results in Fig. 4F and G show that K6PC-5-induced expression of NQO1 mRNA and H01 mRNA were largely inhibited by SphK1 shRNA (Seq-a). K6PC-5-induced upregulations of NQO1, HO1, and GCLC proteins were also inhibited (Fig. 4H). These results suggest that SphK1 is required for K6PC-5-induced Nrf2 activation in SH-SY5Y cells. In the primary murine hippocampal neurons, K6PC-5 induced upregulation of Nrf2, NQO1, H01, and GCLC proteins (Fig. 4I). These results again confirmed that K6PC-5activates Nrf2 signaling in neuronal cells.

Nrf2 knockdown inhibitsK6PC-5-mediated neuroprotection against OGDR

Further studies showed that exposure of SH-SY5Y cells to OGDR also induced minor Nrf2 activation, Nrf2-dependent mRNAs (NQ01, HO1, and GCLC) were slightly increased in OGDR-treated cells (Fig. 5A-C, p $<0.05$ vs. Mock cells). The Nrf2 mRNA level was unchanged (Fig. 5D). shRNA-mediated knockdown of Nrf2 in SH-SY5Y cells (Fig. 5D) blocked OGDRinduced NQ01, H01, and GCLC mRNA expression (Fig. 5A-C). Compared with SH-SY5Y cells treated with the control shRNA, the OGDR-induced increase in the superoxide level (Fig. 5E) was significantly potentiated in Nrf2-silenced SH-SY5Y cells. Importantly, Nrf2-silenced SH-SY5Y cells were more vulnerable to OGDR, presenting with lower viability (Fig. 5F) and increased cell necrosis (LDH release, Fig. 5G). These results imply that OGDR induced minor but nonetheless significant Nrf2 activation to counteract neuronal cell necrosis. In contrast, Nrf2 knockdown intensified neuronal cell death by OGDR. 


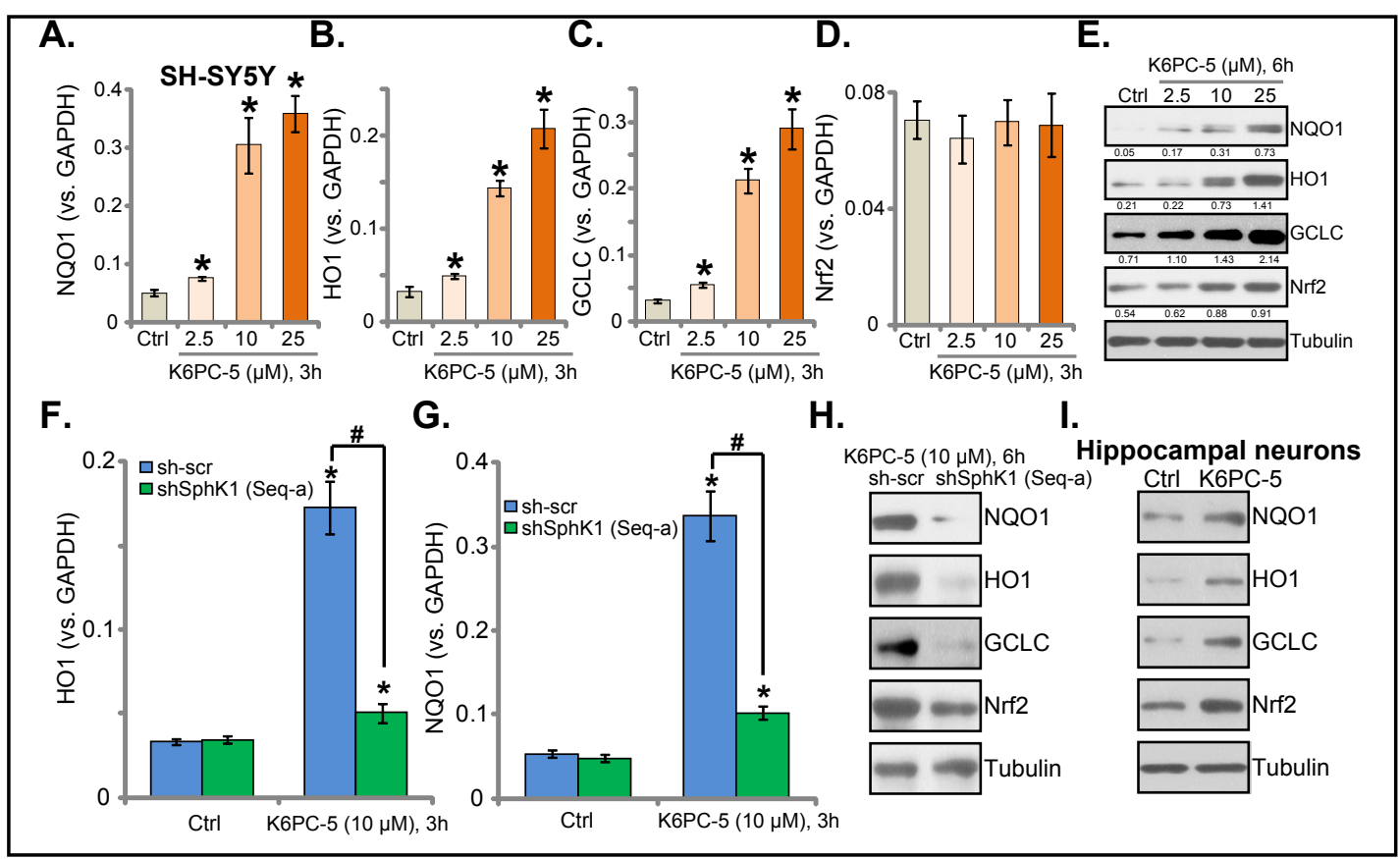

Fig. 4.K6PC-5 activates Nrf2 signaling in neuronal cells. SH-SY5Y cells, with or without lentiviral scrambled control shRNA (sh-scr) or theSphK1 shRNA (Seq-a, sh-SphK1), were treated with the indicated concentrations of K6PC-5 for the indicated times and the mRNA and protein expression levels of the examined Nrf2-related genes were tested by qPCR assay (A-D, F, and G) and western blot ( $E$ and $H$ ), respectively. The primary murine hippocampal neurons were treated with $\mathrm{K} 6 \mathrm{PC}-5(10 \mu \mathrm{M})$ for $6 \mathrm{~h}$, with the expression determined of the listed proteins (I). Quantification of western blot results (E). Bars indicate the mean \pm SD (n=5). ${ }^{*} \mathrm{p}<0.05$ vs. Ctrl. ${ }^{*} \mathrm{p}<0.05$ (F and G). Each experiment was repeated four times and similar results were obtained.

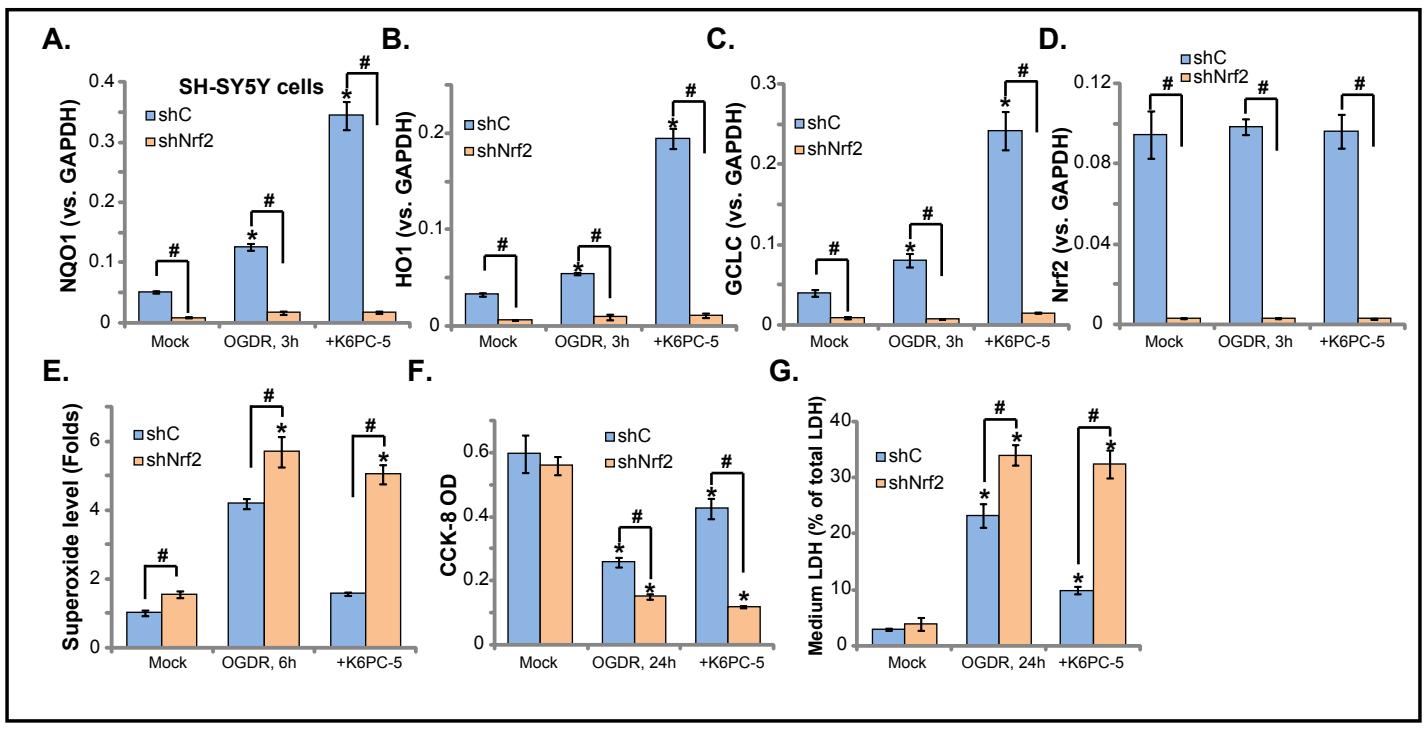

Fig. 5. Nrf2 knockdown inhibits K6PC-5-mediated neuroprotection against OGDR. SH-SY5Y cells, with lentiviral scrambled control shRNA (shC) or Nrf2 shRNA (shNrf2), were treated with K6PC-5 (10 $\mu \mathrm{M})$ and/ or OGDR for the indicated time: the expression levels of the listed mRNAs were examined by qPCR assay (A-D). The superoxide level (E) was also tested, as well as cell viability (CCK-8 assay, F) and necrosis (LDH release assay, G). Bars indicate the mean $\pm \mathrm{SD}(n=5) .{ }^{*} p<0.05$ vs. Mock treatment of shC cells. ${ }^{*} p<0.05$. Each experiment was repeated three times and similar results were obtained. 


\section{Cellular Physiology Cell Physiol Biochem 2018;51:1908-1920

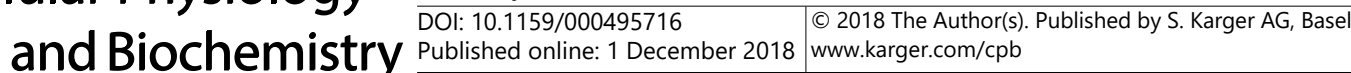 \\ Liu et al.: K6PC-5 Activates SphK1-Nrf2 Signaling}

Notably, K6PC-5 had no effect on the OGDR-induced superoxide increase (Fig. 5E) in Nrf2-silenced SH-SY5Y cells. More importantly, K6PC-5-mediated neuroprotection against OGDR was almost completely nullified by Nrf2 shRNA in SH-SY5Y cells (Fig. 5Fand G). These results suggest that Nrf2 activation is required for K6PC-5-induced anti-OGDR neuroprotective activity.

\section{Discussion}

In this study, we showed that K6PC-5, a SphK1 activator, efficiently protected SH-SY5Y cells and primary hippocampal neurons from OGDR. K6PC-5 activated SphK1, which is required for its activity in neuronal cells, with SphK1 knockdown by targeted shRNA almost completely abolishing K6PC-5-mediated neuroprotection. Considering the upregulation of SphK1 in OGDR-treated cells, our results suggest that SphK1 could be an important neuroprotection target for ischemic injury.

mPTP opening triggers mitochondrial swelling, outer membrane leakage, and Cyto-C release, which have been linked to cell apoptosis alone [37, 38]. However, this theory has recently been challenged. First, apoptosis is an active and ATP-consuming process, but mPTP disruption leads to ATP depletion [11]. Second, Cyp-D knockout mice show normal development, indicating that extensive apoptosis during development is not affected in Cyp-D knockout mice [8]. Third, cell apoptosis by pro-apoptotic Bcl-2 proteins is unaffected in Cyp-D-depleted cells [8, 39, 40]. Further studies have shown that in vitro apoptosis is not inhibited in cells with Cyp-D depletion. However, oxidative stress- and calcium overloadinduced in vitro cell necrosis is clearly suppressed [8,11,39-41]. Thus, the current view is that the mitochondrion is the key hub of both apoptosis and necrosis through a mechanism involving the MPTP $[9-11,38]$.

A number of studies have confirmed that programmed necrosis, an active necrosis pathway, mediates cell death by OGDR and oxidative stress $[3,5,19,32]$. OGDR induces ROS production and oxidative stress, which lead to p53 translocation to the mitochondria to form a complex with Cyp-D. The complex then triggers mitochondrial depolarization, mPTP opening, Cyto- $C$ release, and eventually cell necrosis $[3,5,19,32]$. In the present study, we show that treatment with K6PC-5 blocked the programmed necrosis pathway by OGDR in neuronal cells. This could explain the superior neuroprotective activity of this SphK1 activator.

Nrf2 is arguably one of the most important endogenous antioxidant signaling factors [33-36]. Activated Nrf2 splits from its inhibitor protein Keap1, causing its translocation to the cell nucleus. Freed Nrf2 binds to ARE, triggering the transcription and expression of multiple genes, including the endogenous antioxidant genes, phase II detoxification enzymes, and other cellular defensive genes [33-36]. Our results showed that activation of SphK1 by K6PC5 also activated Nrf2 signaling in neuronal cells. K6PC-5 treatment of neuronal cells induced Nrf2 protein stabilization as well as transcription of Nrf2-regulated genes (NQO1, HO1, and GCLC). Importantly, activation of Nrf2 is required for K6PC-5-mediated neuroprotection against OGDR. Nrf2 shRNA not only exacerbated OGDR-induced injury in neuronal cells, but also abolished K6PC-5-mediated neuronal cell protection. Furthermore, SphK1 shRNA in SH-SY5Y cells almost entirely blocked Nrf2 activation by K6PC-5, suggesting that K6PC-5activated SphK1 acts upstream of Nrf2 in neuronal cells. 


\section{Cellular Physiology Cell Physiol Biochem 2018;51:1908-1920

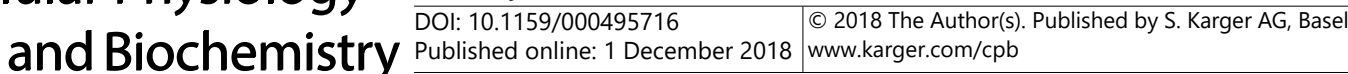

Liu et al.: K6PC-5 Activates SphK1-Nrf2 Signaling

\section{Conclusion}

Taken together, our results suggest that K6PC-5 activates SphK1-Nrf2 signaling to protect neuronal cells from OGDR.

\section{Acknowledgements}

This study was supported by Clinical and Basic Researches of Brain Disease (KYC004), the National Natural Science Foundation of China (81502162), the Science Foundation of Wannan Medical College (WK2017F04) and Kunshan Science Project (KS1644).

\section{Disclosure Statement}

The authors declare no conflicts of interest.

\section{References}

1 Verklan MT: The chilling details: hypoxic-ischemic encephalopathy. J Perinat Neonatal Nurs 2009;23:59-68; quiz 69-70.

2 Allen CL, Bayraktutan U: Oxidative stress and its role in the pathogenesis of ischaemic stroke. Int J Stroke 2009;4:461-470.

-3 Zhao LP, Ji C, Lu PH, Li C, Xu B, Gao H: Oxygen glucose deprivation (OGD)/re-oxygenation-induced in vitro neuronal cell death involves mitochondrial cyclophilin-D/P53 signaling axis. Neurochem Res 2013;38:705713.

4 Gu DM, Lu PH, Zhang K, Wang X, Sun M, Chen GQ, Wang Q: EGFR mediates astragaloside IV-induced Nrf2 activation to protect cortical neurons against in vitro ischemia/reperfusion damages. Biochem Biophys Res Commun 2015;457:391-397.

5 Almeida A, Delgado-Esteban M, Bolanos JP, Medina JM: Oxygen and glucose deprivation induces mitochondrial dysfunction and oxidative stress in neurones but not in astrocytes in primary culture. J Neurochem 2002;81:207-217.

6 Zhao H, Mitchell S, Ciechanowicz S, Savage S, Wang T, Ji X, Ma D: Argon protects against hypoxic-ischemic brain injury in neonatal rats through activation of nuclear factor (erythroid-derived 2)-like 2. Oncotarget 2016;7:25640-25651.

7 Blokhina 0, Virolainen E, Fagerstedt KV: Antioxidants, oxidative damage and oxygen deprivation stress: a review. Ann Bot 2003;91 Spec No:179-194.

8 Baines CP, Kaiser RA, Purcell NH, Blair NS, Osinska H, Hambleton MA, Brunskill EW, Sayen MR, Gottlieb RA, Dorn GW, Robbins J, Molkentin JD: Loss of cyclophilin D reveals a critical role for mitochondrial permeability transition in cell death. Nature 2005;434:658-662.

$>9$ Halestrap AP, Gillespie JP, O’Toole A, Doran E: Mitochondria and cell death: a pore way to die? Symp Soc Exp Biol 2000;52:65-80.

10 Halestrap AP: Calcium, mitochondria and reperfusion injury: a pore way to die. Biochem Soc Trans 2006;34:232-237.

11 Halestrap A: Biochemistry: a pore way to die. Nature 2005;434:578-579.

12 Mullen TD, Obeid LM: Ceramide and apoptosis: exploring the enigmatic connections between sphingolipid metabolism and programmed cell death. Anticancer Agents Med Chem 2012;12:340-363.

13 Vadas M, Xia P, McCaughan G, Gamble J: The role of sphingosine kinase 1 in cancer: oncogene or nononcogene addiction? Biochim Biophys Acta 2008;1781:442-447.

14 Shida D, Takabe K, Kapitonov D, Milstien S, Spiegel S: Targeting SphK1 as a new strategy against cancer. Curr Drug Targets 2008;9:662-673. 


\section{Cellular Physiology Cell Physiol Biochem 2018;51:1908-1920 \begin{tabular}{ll|l} 
DOl: 10.1159/000495716 & 2018 The Author(s). Published by S. Karger AG, Basel \\
and Biochemistry & $\begin{array}{l}\text { Published online: } 1 \text { December } 2018 \\
\text { www.karger.com/cpb }\end{array}$ \\
\cline { 2 - 3 }
\end{tabular}}

Liu et al.: K6PC-5 Activates SphK1-Nrf2 Signaling

15 Kwon YB, Kim CD, Youm JK, Gwak HS, Park BD, Lee SH, Jeon S, Kim BJ, Seo YJ, Park JK, Lee JH: Novel synthetic ceramide derivatives increase intracellular calcium levels and promote epidermal keratinocyte differentiation. J Lipid Res 2007;48:1936-1943.

16 Hong JH, Youm JK, Kwon MJ, Park BD, Lee YM, Lee SI, Shin DM, Lee SH: K6PC-5, a direct activator of sphingosine kinase 1, promotes epidermal differentiation through intracellular Ca2+ signaling. J Invest Dermatol 2008;128:2166-2178.

17 Ji F, Mao L, Liu Y, Cao X, Xie Y, Wang S, Fei H: K6PC-5, a novel sphingosine kinase 1 (SphK1) activator, alleviates dexamethasone-induced damages to osteoblasts through activating SphK1-Akt signaling. Biochem Biophys Res Commun 2015;458:568-575.

18 Petit A, Kawarai T, Paitel E, Sanjo N, Maj M, Scheid M, Chen F, Gu Y, Hasegawa H, Salehi-Rad S, Wang L, Rogaeva E, Fraser P, Robinson B, St George-Hyslop P, Tandon A: Wild-type PINK1 prevents basal and induced neuronal apoptosis, a protective effect abrogated by Parkinson disease-related mutations. J Biol Chem 2005;280:34025-34032.

19 Zheng K, Sheng Z, Li Y, Lu H: Salidroside inhibits oxygen glucose deprivation (OGD)/re-oxygenationinduced $\mathrm{H} 9 \mathrm{c} 2$ cell necrosis through activating of Akt-Nrf2 signaling. Biochem Biophys Res Commun 2014;451:79-85.

20 Qin LS, Jia PF, Zhang ZQ, Zhang SM: ROS-p53-cyclophilin-D signaling mediates salinomycin-induced glioma cell necrosis. J Exp Clin Cancer Res 2015;34:57.

21 Vaseva AV, Marchenko ND, Ji K, Tsirka SE, Holzmann S, Moll UM: p53 opens the mitochondrial permeability transition pore to trigger necrosis. Cell 2012;149:1536-1548.

22 Wu X, Liu D, Gao X, Xie F, Tao D, Xiao X, Wang L, Jiang G, Zeng F: Inhibition of BRD4 Suppresses Cell Proliferation and Induces Apoptosis in Renal Cell Carcinoma. Cell Physiol Biochem 2017;41:1947-1956.

-23 Wang Y, Liu J, Tao Z, Wu P, Cheng W, Du Y, Zhou N, Ge Y, Yang Z: Exogenous HGF Prevents Cardiomyocytes from Apoptosis after Hypoxia via Up-Regulating Cell Autophagy. Cell Physiol Biochem 2016;38:2401-2413.

24 Brooks MM, Neelam S, Fudala R, Gryczynski I, Cammarata PR: Lenticular mitoprotection. Part A: Monitoring mitochondrial depolarization with JC-1 and artifactual fluorescence by the glycogen synthase kinase-3beta inhibitor, SB216763. Mol Vis 2013;19:1406-1412.

25 Yao C, Wu S, Li D, Ding H, Wang Z, Yang Y, Yan S, Gu Z: Co-administration phenoxodiol with doxorubicin synergistically inhibit the activity of sphingosine kinase-1 (SphK1), a potential oncogene of osteosarcoma, to suppress osteosarcoma cell growth both in vivo and in vitro. Mol Oncol 2012;6:392-404.

26 Gong YQ, Huang W, Li KR, Liu YY, Cao GF, Cao C, Jiang Q: SC79 protects retinal pigment epithelium cells from UV radiation via activating Akt-Nrf2 signaling. Oncotarget 2016;7:60123-60132.

27 Ji C, Huang JW, Xu QY, Zhang J, Lin MT, Tu Y, He L, Bi ZG, Cheng B: Gremlin inhibits UV-induced skin cell damages via activating VEGFR2-Nrf2 signaling. Oncotarget 2016;7:84748-84757.

28 Lu PH, Chen MB, Liu YY, Wu MH, Li WT, Wei MX, Liu CY, Qin SK: Identification of sphingosine kinase 1 (SphK1) as a primary target of icaritin in hepatocellular carcinoma cells. Oncotarget 2017;8:22800-22810.

29 Zhang L, Yu H, Sun Y, Lin X, Chen B, Tan C, Cao G, Wang Z: Protective effects of salidroside on hydrogen peroxide-induced apoptosis in SH-SY5Y human neuroblastoma cells. Eur J Pharmacol 2007;564:18-25.

30 Zhang D, Zhang JJ, Liu GT: The novel squamosamide derivative FLZ protects against 6-hydroxydopamineinduced apoptosis through inhibition of related signal transduction in SH-SY5Y cells. Eur J Pharmacol 2007;561:1-6.

-31 Shao JJ, Peng Y, Wang LM, Wang JK, Chen X: Activation of SphK1 by K6PC-5 Inhibits Oxygen-Glucose Deprivation/Reoxygenation-Induced Myocardial Cell Death. DNA Cell Biol 2015;34:669-676.

-32 Zheng K, Zhang Q Lin G, Li Y, Sheng Z, Wang J, Chen L, Lu HH: Activation of Akt by SC79 protects myocardiocytes from oxygen and glucose deprivation (OGD)/re-oxygenation. Oncotarget 2017;8:1497814987.

33 Krajka-Kuzniak V, Paluszczak J, Baer-Dubowska W: The Nrf2-ARE signaling pathway: An update on its regulation and possible role in cancer prevention and treatment. Pharmacol Rep 2017;69:393-402.

-34 Done AJ, Traustadottir T: Nrf2 mediates redox adaptations to exercise. Redox Biol 2016;10:191-199.

-35 Zhang H, Davies KJ, Forman HJ: Oxidative stress response and Nrf2 signaling in aging. Free Radic Biol Med 2015;88:314-336.

-36 Vriend J, Reiter RJ: The Keap1-Nrf2-antioxidant response element pathway: a review of its regulation by melatonin and the proteasome. Mol Cell Endocrinol 2015;401:213-220. 


\section{Cellular Physiology Cell Physiol Biochem 2018;51:1908-1920 \begin{tabular}{ll|l} 
and Biochemistry & $\begin{array}{l}\text { DOl: 10.1159/000495716 } \\
\text { Published online: T December } 2018\end{array}$ & $\begin{array}{l}\text { () } 2018 \text { The Author(s). Published by S. Karger AG, Basel } \\
\text { www.karger.com/cpb }\end{array}$ \\
\hline
\end{tabular}}

Liu et al.: K6PC-5 Activates SphK1-Nrf2 Signaling

-37 Javadov S, Kuznetsov A: Mitochondrial permeability transition and cell death: the role of cyclophilin d. Front Physiol 2013;4:76.

38 Halestrap AP, McStay GP, Clarke SJ: The permeability transition pore complex: another view. Biochimie 2002;84:153-166.

-39 Schinzel AC, Takeuchi O, Huang Z, Fisher JK, Zhou Z, Rubens J, Hetz C, Danial NN, Moskowitz MA, Korsmeyer SJ: Cyclophilin D is a component of mitochondrial permeability transition and mediates neuronal cell death after focal cerebral ischemia. Proc Natl Acad Sci U S A 2005;102:12005-12010.

-40 Nakagawa T, Shimizu S, Watanabe T, Yamaguchi O, Otsu K, Yamagata H, Inohara H, Kubo T, Tsujimoto Y: Cyclophilin D-dependent mitochondrial permeability transition regulates some necrotic but not apoptotic cell death. Nature 2005;434:652-658.

-41 Basso E, Fante L, Fowlkes J, Petronilli V, Forte MA, Bernardi P: Properties of the permeability transition pore in mitochondria devoid of Cyclophilin D. J Biol Chem 2005;280:18558-18561. 